\title{
Robot-assisted donor hysterectomy in uterus transplantation - a modality to increase reproducibility
}

 \\ ${ }^{1}$ Department of Obstetrics and Gynaecology, Second Faculty of Medicine, Charles University and Motol University Hospital, \\ Prague, Czech Republic \\ ${ }^{2}$ Faculty of Medicine in Plzeň, Charles University, Czech Republic
}

\begin{abstract}
Uterus transplantation is a non-lifesaving vascularized composite allotransplantation procedure requiring immunosuppression until removal of the graft. The focus of uterus transplantation is changing regarding refining individual treatment procedures included in this complex treatment of absolute uterine factor infertility, such as robot-assisted donor hysterectomy. The inferior hypogastric nerve plexus should be preserved during robotic dissection of the ureter and uterine vessels to prevent postoperative complications such as urine and fecal evacuation disturbances and sexual disorders. As most uterus transplantations have been performed in living donor concepts, robot-assisted donor hysterectomy should contribute to increased availability of uterus transplantation, particularly because it uses the precise blood-less technique of surgical dissection in the deep pelvis and has cosmetic benefits among living donors.
\end{abstract}

Key words: robot-assisted surgery; hysterectomy; uterus; transplantation; living donor.

Ginekologia Polska 2021; 92, 7: 528-531

\section{INTRODUCTION}

Absolute uterine factor infertility (AUFI) due to an absent or non-functional uterus has been a long-neglected form of female infertility highlighted at the beginning of the $21^{\text {st }}$ century by Swedish researchers in pioneering studies on animal and human uterine transplantation (UTX) [1, 2]. UTx is a temporary vascularized composite allotransplantation technique requiring immunosuppressive therapy until removal of the graft (after cesarean deliveries or approximately up to five posttransplant years). As adoption and gestational surrogacy are not available in many countries worldwide owing to legal, financial, ethical, religious, and social reasons, UTx could be a solution for infertile women with congenital or acquired AUFI. Although 20 years have passed since the first human UTx attempt in Saudi Arabia [3], and eight years since the onset of the first human living donor UTx study in Sweden [2], the overall number of UTx procedures performed worldwide is still only around 70. Thus, UTx seems to be a low-volume transplant procedure in the future. However, approximately 20 children born from transplanted uteri over the last six years indicate the potential of this infertility treatment.

The focus of UTx research is changing with respect to refining procedures associated with this complex treatment of infertility, such as robot-assisted donor hysterectomy. Minimally invasive surgical approaches using conventional or robot-assisted laparoscopy play an important role in gynecologic surgery, particularly in oncological treatment and surgery for complicated endometriosis. The first ever laparoscopic-assisted retrievals of uterus in two living donors (mothers) and the successful transplantations to recipients (daughters) were performed by Puntambekar et al. in Pune, India in 2017 [4, 5]. Similarly, to conventional laparoscopy performed by skilled surgeon, the technical advantage of robot-assisted surgery is the use of a 3-dimensional camera and instruments that allow for both fine tissue dissection and optimal surgical access to the deep pelvis. Based on the experience with both UTx and oncologic operations in gynecology [6-8], in this article, we discuss robot-assisted donor hysterectomy with regard to minimizing surgical

\footnotetext{
Corresponding author:

Roman Chmel Jr.

Department of Obstetrics and Gynaecology, Second Faculty of Medicine, Charles University and Motol University Hospital, Prague, Czech Republic; Faculty of Medicine in Plzeň, Charles University, Czech Republic

e-mail: roman.chmel@fnmotol.cz

This article is available in open access under Creative Common Attribution-Non-Commercial-No Derivatives 4.0 International (CC BY-NC-ND 4.0) license, allowing to download articles and share them with others as long as they credit the authors and the publisher, but without permission to change them in any way or use them commercially.
} 
morbidity and invasiveness in living donors of the uterus due to the less traumatic dissection of the vascular and other anatomical structures in the retroperitoneal space, particularly concerning the tedious dissection of the uterine veins to isolate them from the firm attachments to paracervical tissue and ureters.

\section{MAIN ASPECTS OF UTERINE GRAFT PROCUREMENT}

No serious surgical problems were reported in previous acquisitions of the uterine arteries during living donor hysterectomies using the open approach $[2,6]$. This aspect of uterine graft procurement is well known to gynecologists who are specialized in radical hysterectomy, regardless of the abdominal, laparoscopic, or robotic nature of cervical cancer surgery. At the beginning of the uterine graft retrieval, the paravesical and the medial and lateral pararectal spaces are opened after division of the round ligaments. Next, the large flap of the bladder peritoneum is dissected for the fixation of the uterus in the recipient. Thereafter, the bladder is dissected from the cervix and vagina. Dissection of the common and internal iliac arteries up to the area of the obliterated umbilical artery precedes the dissection of the uterine artery between its exit from the internal iliac artery up to the uterine edge. Internal iliac arteries should be preserved in living donors of the uterus to avoid the risk of postoperative gluteal claudication after ligation. To ensure optimal blood inflow to the graft, patches of the internal iliac vessels (or parts of their anterior branches) should be utilized when harvesting the uterine arteries $[6,9,10]$.

Pre-procurement insertion of ureteral stents is helpful but optional. The ureter should be dissected up to the parametrial ureteric channel and its inlet into the urinary bladder. During open procurement surgery, the uterine veins are poorly visible without magnifying loupes or a surgical microscope. A skilled surgeon experienced in microsurgical techniques dissects these small veins up to their inlet in the internal iliac vessels. However, dissection of the uterine veins is technically challenging as they may have individual variations and over- and under-ride the ureters. The uterine vein is usually created from two to three veins that come from the uterine body and converge into a common uterine vein or have separate small inlets to the internal iliac vein. Separation of the uterine vein from the vaginal cuff and the ureter between its inlet to the bladder and the crossing with the uterine artery is usually the most time-consuming part of graft procurement. Separation is difficult to perform using both open and robot-assisted surgical techniques, particularly because of the variations in the uterine veins. In contrast, separation of the ovarian veins (or their utero-ovarian segments) that can be utilized for venous outflow from the transplanted uterus is technically less complicated and, as such, usually faster than the complex dissection and retrieval of the uterine veins.

After the uterus is completely mobilized from the adjacent tissues and organs and the uterine vasculature is dissected, the vagina is transected approximately $15 \mathrm{~mm}$ caudal to the vaginal vault. Thereafter, the internal iliac vessels are clamped, uterine vessels are divided, and, finally, the uterus is removed from the donor.

\section{THE POTENTIAL ADVANTAGES OF ROBOT- -ASSISTED DONOR HYSTERECTOMY}

Using open, laparoscopic, and robot-assisted nerve-sparing radical hysterectomies, the hypogastric and pelvic splanchnic nerves as well as the pelvic plexus with vesical branches can be spared [8]. The inferior hypogastric nerve plexus can be preserved during laparoscopic dissection of the ureters, uterine arteries, and utero-sacral ligaments to avoid postoperative complications such as urinary and fecal evacuation disturbances and sexual disorders. This can be also done during laparotomy using surgical loupes. One Czech living uterus donor was found to have prolonged bladder hypotonia immediately after open procurement surgery requiring three months of suprapubic drainage and bladder training to reach normal spontaneous micturition without residual urine [6]. As both laparoscopic and robot-assisted retrieval surgery allows for good visibility of the nerve structures in the deep pelvis like surgical microscope in open surgery, laparoscopic and robot-assisted techniques of nerve-sparing dissection can be used to avoid damage to the above nerve structures.

Most previous open uterus retrievals were performed with minimal tissue trauma and without endangering the adjacent anatomical structures in the parametria. However, both robot-assisted and conventional laparoscopic procurement performed by an experienced surgeon might simplify the dissection of the uterine vessels between the internal iliac vessels and uterine edges as well as the dissection of the ureters. Both approaches show the same results. The laparoscopic dissection of the connective tissues, vessels, and ureters in the deep pelvis is safer than the laparotomy approach when performed by skilled surgeons trained in minimally invasive operative techniques, particularly because of the excessive use of diathermy dissection in the retroperitoneal space when performing open procurement surgeries $[2,6]$.

In addition to the above advantages of the laparoscopic approach, the comfort of the surgeon is an important benefit of the robot-assisted surgery. Comfortable sitting position with appropriate setting of individually coded robotic console is convenient particularly in long procedures such as radical hysterectomy with lymphadenectomy for cervical cancer and procurement of uterus in living donor. 
However, robot-assisted surgery has also several limitations, particularly time-consuming setting of robotic surgical system which can be shortened with the number of performed procedures; use of complex instruments that increase the overall cost of UTx; and 3-dimensional visualization that can cause surgeon's discomfort (e.g., headache), especially when using robot-assisted technique daily and not alternating between different surgical approaches on a regular basis.

\section{FULLY ROBOTIC AND COMBINED ROBOTIC/ /OPEN APPROACHES IN ROBOT-ASSISTED DONOR HYSTERECTOMY}

As the main purpose of robot-assisted approach is a less traumatic, more precise, and blood-less surgery than conventional methods, fully robot-assisted donor hysterectomy seems to be a better option than the combined option. Moreover, to minimize the burden on living donors, the uterus should be extracted through the vagina in a sterile bag to prevent bacterial contamination, as observed in the first Chinese robot-assisted uterus procurement for the subsequent transplantation in 2015 and five robot-assisted donor surgeries performed recently in the USA [11, 12]. Of course, contusion of the uterus should be avoided when transvaginal removal of the graft is the preferred option. However, a combined robotic/open approach to donor hysterectomy involving robotic dissection of the uterine vessels and mobilization of all vascular and nerve structures in the parametria including ureters followed by the subsequent removal of the uterine graft after conversion to open surgery, as has been performed in 2017-2019 in Sweden, should also be justified, particularly during the learning-curve period $[13,14]$. The Swedish robot-assisted retrieval surgeries showed gradually reducing durations throughout the trial. The last operation had the shortest duration, which was still 10 hours. The length of their donor surgeries was based particularly on the study protocol to separate both uterine veins to be prepared for anastomosis to the internal iliac vessels as the main drainage option from the transplanted uterus. The proximal parts of the utero-ovarian veins were harvested to be used only for the salvage outflow when the uterine veins were of poor quality and seemed unusable.

In five fully robotic retrievals of the uterus in the USA, the study protocol required harvesting of the proximal parts of both utero-ovarian veins together with both uterine veins. However, only 5 out of 10 uterine veins were successfully harvested [12]. Moreover, only 2 out of 5 harvested uterine veins were used for the anastomosis to external iliac veins in the recipient, while all 10 utero-ovarian veins were utilized for the graft's venous outflow. While in the Swedish donors of the uterus, both uterine veins were successfully harvested in all eight retrievals, the team from the USA probably had some technical difficulties that led to only $50 \%$ success of robotic dissection of the uterine veins. As live birth after UTx without the outflow via utero-ovarian veins has been reported only by the Dallas research team [15], complete surgical as well as reproductive data of further UTx studies with different venous outflows will shed light on the optimal venous drainage from the transplanted uterus.

While the cosmetic reasons for the preference of robot-assisted hysterectomy in living donors of the uterus are obvious, the prevalence of both ureteric lesions in three (23\%) and reversible pressure alopecia (very likely due to duration of the surgery) in two donors (15\%) out of 13 women who underwent robotic retrievals of the uterus in Sweden and the USA seems relatively high [12,14]. Further experience with robot-assisted retrieval of the uterus for transplantation is required to identify the risk factors associated with urological complications of donor hysterectomy.

\section{CONCLUSION}

Moving UTx to the clinical setting requires comprehensive practical guidelines to ensure a high degree of consistency and safety. If the results of the pioneering studies on robot-assisted donor hysterectomy confirm satisfactory surgical and reproductive results, this less-invasive procurement option should become preferable for the majority of living donors of the uterus. However, further research is needed to confirm the applicability and safety of the robot-assisted procurement of the uterus in clinical practice.

\section{Conflict of interest}

The authors declare that they have no conflicts of interest regarding the publication of this article.

\section{Contributions of authors}

We confirm that all the co-authors have been included, have contributed to the final manuscript, and have approved it. $\mathrm{RCjr}$ and RC designed the study, analyzed the data, and wrote the manuscript. ZP and MN designed the study and critically reviewed the manuscript.

\section{Acknowledgments}

The preparation of this manuscript was supported by the Ministry of Health, Czech Republic, Conceptual Development of Research Organization, Motol University Hospital, Prague, Czech Republic 00064203.

\section{REFERENCES}

1. Brännström M, Diaz-Garcia C, Hanafy A, et al. Uterus transplantation: animal research and human possibilities. Fertil Steril. 2012; 97(6): 1269-1276, doi: 10.1016/j.fertnstert.2012.04.001, indexed in Pubmed: 22542990.

2. Brännström $M$, Johannesson $L$, Dahm-Kähler $P$, et al. First clinical uterus transplantation trial: a six-month report. Fertil Steril. 2014; 101(5): 1228-1236, doi: 10.1016/j.fertnstert.2014.02.024, indexed in Pubmed: 24582522 
3. Fageeh $\mathrm{W}$, Raffa $\mathrm{H}$, Jabbad $\mathrm{H}$, et al. Transplantation of the human uterus. International Journal of Gynecology \& Obstetrics. 2002; 76(3): 245-251, doi: 10.1016/s0020-7292(01)00597-5.

4. Puntambekar S, Telang M, Kulkarni P, et al. Laparoscopic-Assisted Uterus Retrieval From Live Organ Donors for Uterine Transplant. J Minim Invasive Gynecol. 2018; 25(4): 571-572, doi: 10.1016/j.jmig.2017.11.001, indexed in Pubmed: 29133152.

5. Puntambekar S, Telang M, Kulkarni P, et al. Laparoscopic-Assisted Uterus Retrieval From Live Organ Donors for Uterine Transplant: Our Experience of Two Patients. J Minim Invasive Gynecol. 2018; 25(4): 622-631, doi: 10.1016/j.jmig.2018.01.009, indexed in Pubmed: 29366966.

6. Chmel R, Novackova M, Janousek $L$, et al. Revaluation and lessons learned from the first 9 cases of a Czech uterus transplantation trial: Four deceased donor and 5 living donor uterus transplantations. Am J Transplant. 2019; 19(3): 855-864, doi: 10.1111/ajt.15096, indexed in Pubmed: 30151893.

7. Chmel R, Pastor Z, Matecha J, et al. Uterine transplantation in an era of successful childbirths from living and deceased donor uteri: Current challenges. Biomed Pap Med Fac Univ Palacky Olomouc Czech Repub. 2020; 164(1): 115-120, doi: 10.5507/bp.2019.020, indexed in Pubmed: 31132074.

8. Novackova M, Pastor Z, Chmel R, et al. Urinary tract morbidity after nerve-sparing radical hysterectomy in women with cervical cancer. Int Urogynecol J. 2020; 31(5): 981-987, doi: 10.1007/s00192-019-04083-9, indexed in Pubmed: 31444536.
9. Brännström M, Dahm-Kähler P, Kvarnström N. Robotic-assisted surgery in live-donor uterus transplantation. Fertil Steril. 2018; 109(2): 256-257, doi: 10.1016/j.fertnstert.2017.12.007, indexed in Pubmed: 29395094.

10. Fornalik H, Fornalik N. Uterus transplantation:robotic surgeon perspective. Fertil Steril. 2018; 109(2): 365, doi: 10.1016/j.fertnstert.2017.10.038, indexed in Pubmed: 29246556.

11. Wei Li, XueT, Tao KS, et al. Modified human uterus transplantation using ovarian veins for venous drainage: the first report of surgically successful robotic-assisted uterus procurement and follow-up for 12 months. Fertil Steril. 2017; 108(2): 346-356.e1, doi: 10.1016/j.fertnstert.2017.05.039, indexed in Pubmed: 28778283.

12. Johannesson L, Koon EC, Bayer J, et al. Dallas UtErus Transplant Study: Early Outcomes and Complications of Robot-assisted Hysterectomy for Living Uterus Donors. Transplantation. 2021; 105(1): 225-230, doi: 10.1097/TP.0000000000003211, indexed in Pubmed: 32150040.

13. Brännström $M, D a h m-K a ̈ h l e r P, K v a r n s t r o ̈ m ~ N$, et al. Live birth after robotic-assisted live donor uterus transplantation. Acta Obstet Gynecol Scand. 2020; 99(9): 1222-1229, doi: 10.1111/aogs.13853, indexed in Pubmed: 32196630

14. Brännström $M$, Kvarnström N, Groth K, et al. Evolution of surgical steps in robotics-assisted donor surgery for uterus transplantation: results of the eight cases in the Swedish trial. Fertil Steril. 2020; 114(5): 1097-1107, doi: 10.1016/j.fertnstert.2020.05.027, indexed in Pubmed: 32828495.

15. Testa G, McKenna GJ, Gunby RT, et al. First live birth after uterus transplantation in the United States. Am J Transplant. 2018; 18(5): 1270-1274, doi: 10.1111/ajt.14737, indexed in Pubmed: 29575738. 